subgroups. Participants completed validated, age-appropriate questionnaires examining standard psychological parameters. Participants also underwent an evaluation of exercise, including formal exercise stress testing and measurement of free-living activity using an ActiGraph accelerometer. Results were analysed using parametric methods. 143 patients (mean age 15.6 years) consented to participate, 86 were male (60\%) and 105 had major CHD (73\%). Diagnostic subgroups included 39 acyanotic (27.3\%), 61 acyanotic corrected $(42.7 \%), 30$ cyanotic corrected $(21.0 \%)$ and $13(9 \%)$ cyanotic palliated patients. Beck Youth Inventory demonstrated that individuals with major CHD, particularly cyanotic palliated patients, had higher anxiety scores ( $p$ value $0.01(-8.42,-1.13)$ ). There were no significant differences across study groups for selfesteem or other psychological parameters. 134 participants $(93.7 \%)$ took part in regular exercise each week. There was no significant difference in activity score between study groups. On formal exercise testing, more complex patients performed worse at peak exercise. Exercise time for acyanotic group 11.73 mins (sd 3.74) compared to 8.26 mins (sd 4.08) in cyanotic palliated group, p value $0.002(1.32,5.61))$. However, patients with major CHD had significantly higher activity counts. Correlation analysis showed that selfesteem and health locus of control were important predictor variables for activity. Self-esteem and mood seem well preserved in adolescents with $\mathrm{CHD}$ as a whole. The majority of young people with CHD, in this group, take part in regular exercise. Surprisingly, complex patients rate themselves to be as active as those with minor CHD. While accelerometer data indicate that the group may be more active day to day, they are limited in terms of peak exercise duration. The experience of growing up with a chronic condition may therefore have a positive effect on psychological health and interventions targeted around this area may influence activity.

\section{MUTATIONS IN THE SARCOMERE PROTEIN GENE MYH7 IN EBSTEIN'S ANOMALY}

doi:10.1136/heartjnl-2011-300198.134

${ }^{1} \mathrm{~T}$ Rahman, ${ }^{1} \mathrm{~J}$ Goodship, ${ }^{2} \mathrm{~A}$ Postma, ${ }^{2} \mathrm{~K}$ Engelen, ${ }^{2} \mathrm{~B}$ Mulder, ${ }^{3} \mathrm{~S}$ Klaassen, ${ }^{4} \mathrm{~B}$ Keavney. ${ }^{1}$ Institute of Human Genetics, Newcastle upon Tyne, UK; ${ }^{2}$ Academic Medical Centre, Amsterdam, The Netherlands; ${ }^{3}$ Max-Delbrueck-Center for Molecular Medicine, Berlin, Germany; ${ }^{4}$ Institute of Human Genetics, Newcastle upon Tyne, UK

Background Ebstein's anomaly is a rare congenital heart malformation characterised by adherence of the septal and posterior leaflets of the tricuspid valve to the underlying myocardium. As there have been reports of abnormal left ventricular morphology and function in patients with Ebstein's anomaly we hypothesised that mutations in the $\beta$-myosin heavy chain (MYH7) may be associated with Ebstein's anomaly.

Methods MYH7 mutation analysis was undertaken in 141 unrelated affected individuals with Ebstein's anomaly using next-generation sequencing on the 454 platform. 64 probands had no associated cardiac anomalies. The most common associated cardiac malformation were atrial septal defect (48 probands) and left ventricular non-compaction (LVNC) (7 probands). Where mutations were discovered, family studies were undertaken and the segregation of the mutation with disease was investigated.

Results Heterozygous mutations were identified in eight of the probands including six of the seven with LVNC. Two patients had the same mutation; of the seven distinct mutations, five were novel (four missense changes and an in-frame deletion) and two have been previously reported in patients with hypertrophic cardiomyopathy. Family studies revealed additional members with LVNC for three of the probands, one of whom also had a relative with Ebstein's anomaly. In these three pedigrees the mutation segregated with disease.

Conclusions Mutations in MYH7 occur relatively frequently in Ebstein's anomaly accompanied by LVNC. This study is another example of mutations in a sarcomere protein causing congenital heart malformation.

\section{GENE SCREENING OF THE SECONDARY HEART FIELD NETWORK IN TETRALOGY OF FALLOT PATIENTS}

doi:10.1136/heartjnl-2011-300198.135

A Töpf, H R Griffin, D H Hall, E Glen, B D Keavney, J A Goodship; The Change Study Collaborators. Institute of Human Genetics, Newcastle upon Tyne, UK

Background Tetralogy of Fallot (TOF) is the most common cyanotic heart defect, affecting 3-6 infants for every 10000 births. TOF is phenotypically well defined; it consists of four heart abnormalities: a VSD, an over-riding aorta, a narrowed pulmonary valve and right ventricular hypertrophy. During heart development two heart fields can be distinguished. The first one gives origin to the left ventricle and contributes to the right and left atria. The secondary heart field gives origin to the right ventricle and the outflow tract. Each of these fields can be identified by the expression of specific markers. As TOF is a malformation of the outflow tract, we hypothesised genes involved in the regulatory network of the secondary heart field were particularly good candidates for TOF susceptibility.

Methods We examined by standard Sanger method the full exonic and intron boundary regions of 14 secondary heart field genes, namely NKX2-5, GATA4, TBX20, MEF2C, BOP, HAND2, FOXC1, FOXC2, TBX1, FOXA2, FGF10, FGF8, ISL1 and FOXH1, in a panel of 93 TOF patients. All newly discovered rare variants were checked in a panel of 1000 control chromosomes by multiplex Sequenom assays. When available, parents of cases were screened to assess inheritance of the rare variant.

Results We re-sequenced a total of 80 exons and $\sim 30 \mathrm{~Kb}$. Among the 14 genes studied we found a total of 50 new variants, of which 23 were exclusive to the patient population, ie, were absent from 1000 normal chromosomes. Nine of these variants cause change in the aminoacid sequence. We found a functional 19aa deletion of a highly conserved region of TBX1. In FOXC1 we found a contraction of both alanine and glycine tracts. An alanine expansion, usually known to be deleterious, was found in HAND2. Four non-synonymous changes were found in FOXA2. Most patients presented just one variant, however 3 patients presented two, and one patient presented up to 3 variants. All patients were heterozygotes for the variants, and had inherited them from one of their phenotypically normal parents (when parental information was available). In addition, $75 \%$ of the variants were inherited from the mother.

Conclusions Although genes of the secondary heart field seemed good candidates for TOF susceptibility, thus far we have not found any strong indication of unique causal effect, as all variation found in probands was also present in their unaffected parents. However, the presence of multiple variants in the same proband may result in the disruption of gene-gene interactions in the secondary heart field pathway, which in turn may lead to outflow tract defects. Based on our results, it would seem more likely that susceptibility to TOF be determined by a larger number of small genetic contributions which are also modified by environmental factors. It is evident that larger scale analysis of significant numbers of whole genomes/exomes will be necessary to better understand the molecular aetiology of TOF.

\section{6 SHOULD FAMILIAL SCREENING BE ROUTINELY OFFERED TO PATIENTS WITH BICUSPID AORTIC VALVE DISEASE?}

doi:10.1136/heartjnl-2011-300198.136

R Panayotova, S Hosmane, A Macnab, P Waterworth. University Hospital of South Manchester, Manchester, UK

Background Bicuspid aortic valve (BAV) disease is one of the most common congenital cardiac abnormalities with prevalence in the 
general population of up to $2 \%$. There has been growing evidence supporting its familial predisposition with an autosomal dominant pattern of inheritance. It is often associated with ascending aortic dilatation and dissection, occurring at a younger age than in patients with idiopathic aortic aneurysms. BAV disease carries a 6\% lifetime risk of aortic dissection, 9 times higher than that of the general population. Thus, the presence of BAV and dilatation of the ascending aorta requires regular monitoring with a view to timely pre-emptive surgery. Current ACC/AHA guidelines state that echocardiographic screening of first degree relatives of patients with BAV is recommended. This, however, to our knowledge, is not routinely done within the UK.

Methodology and Results We set out to explore the practicalities of running a routine echocardiographic screening programme for first degree relatives of patients with BAV disease. We identified a total of 47 patients who had undergone aortic valve surgery performed by the same Consultant Cardiothoracic Surgeon in the context of BAV disease in the period May 2007-September 2009. Screening of first degree relatives was offered to these patients. 24 patients (51\%) gave us information regarding family members who would like to attend for an echocardiogram. A total of 75 first degree relatives were referred-an approximate average of 3 per patient. Out of these, 52 relatives $(70 \%)$ actually attended for an appointment. The remainder did not undergo testing with us as they either lived in a different geographic region or expressed a personal preference not to be scanned at this time. The incidence of newly diagnosed bicuspid aortic valve disease in our cohort of first degree relatives was $8 \%$ (4 out of 52 relatives). One of these asymptomatic individuals had a significant ascending aortic aneurysm, which required prompt surgery. Among the relatives of the 24 index patients, there were a total of 8 cases (3: 1 ratio) of bicuspid aortic valve disease-either known or newly diagnosed via screening.

Conclusions There is a relatively high prevalence and incidence of bicuspid aortic valve disease among first degree relatives of patients with this common congenital cardiac abnormality. Routine echocardiographic screening should be offered to these families. Implementing such a programme is limited by adequate motivation to attend for a screening test if well, and by varying clinical practice in different geographic regions. Patients with bicuspid aortic valve disease should be made aware of its familial pattern of inheritance and screening of their first degree relatives should be actively pursued in order to reduce the potential morbidity and mortality associated with this condition and its related aortopathy.

\section{A CITED2->VEGFA PATHWAY COUPLES MYOCARDIAL AND CORONARY VASCULAR GROWTH IN THE DEVELOPING MOUSE HEART}

doi:10.1136/heartjnl-2011-300198.137

${ }^{1} S$ D Bamforth, 'S T MacDonald, 'J Braganca, ${ }^{1} \mathrm{C}-\mathrm{M}$ Chen, ${ }^{1} \mathrm{C}$ Broadbent, ${ }^{1} \mathrm{~J}$ E Schneider, ${ }^{2} \mathrm{R}$ Schwartz, ${ }^{1} \mathrm{~S}$ Bhattacharya. 'University of Oxford, Oxford, UK; ${ }^{2}$ Texas A\&M Health Science Centre, Houston, Texas, USA

Introduction Myocardial development is dependent on the concomitant growth of cardiomyocytes and a supporting vascular network. The coupling of myocardial and coronary vascular development is mediated in part by VEGFA signalling. Cited2 is a transcriptional cofactor that can inhibit hypoxia-activated transcription and also acts as a co-activator for transcription factors such as TFAP2. Genetic evidence indicates that Cited 2 is essential for cardiac left-right patterning via regulation of the Nodal-Pitx2c left-right patterning pathway. Zygotic and epiblastic deletion of Cited2 results in atrioventricular septation, outflow tract and aortic arch abnormalities, as well as left-right patterning defects such as right-isomerism. Cited2 is also essential for adrenal, neural crest, liver, lung, lens and placental development. However, the early requirement of Cited 2 in left-right patterning and placental development makes it difficult to identify a later specific role for Cited 2 in myocardial development. To overcome this problem we therefore investigated the role of Cited2 in the myocardium by conditional deletion in cardiomyocyte precursors. Methods Cited 2 was selectively deleted from cardiomyocytes by intercrossing mice transgenic for Cited 2 and Nkx2-5Cre. Embryos were collected and processed for analysis by histology, MRI, X-Gal staining, quantitative reverse transcriptase PCR (Q-RTPCR), chromatin immunoprecipitation and transient transfection assays.

Results The cardiomyocyte specific knockout of Cited 2 results in abnormal myocardial compact zone growth and ventricular septal defects. This is associated with a decreased ratio in the number of small vessels to large vessels, and a reduction in Vegfa expression. We also show that CITED2 is present at the Vegfa promoter in mouse embryonic hearts, and that it stimulates human VEGFA promoter activity in cooperation with TFAP2 transcription factors in transient transfection assays. However, we observed no change in the myocardial expression of the left-right patterning gene Pitx $2 c$, a known target of Cited2.

Conclusions The myocardial and capillary defects observed in myocardial loss of Cited 2 are not associated with Pitx $2 c$ deficiency and suggests that Cited 2 can cause myocardial and vascular defects via a mechanism that is distinct from its effect on the left-right patterning pathway. Our results delineate a novel mechanism of Vegfa regulation by CITED2 and TFAP2 transcription factors, and indicate that coupling of myocardial and coronary vascular growth in the developing mouse heart occurs, at least in part, through a Cited2->Vegfa pathway. This pathway may be targeted for the treatment of heart failure resulting from ischaemic heart disease.

\section{CELL-SPECIFIC ROLE OF NOX2 NADPH OXIDASE IN DEVELOPMENT OF ANGIOTENSIN II-INDUCED CARDIAC FIBROSIS IN VIVO}

doi:10.1136/heartjnl-2011-300198.138

${ }^{1} \mathrm{~S}$ Chaubey, ${ }^{1} \mathrm{C}$ E Murdoch, ${ }^{1} \mathrm{~A}$ Ivetic, ${ }^{1} \mathrm{~B}$ Yu, ${ }^{2} \mathrm{D}$ Vanhoutte, ${ }^{2} \mathrm{~S}$ Heymans, ${ }^{1} \mathrm{~A}$ Brewer, ${ }^{1} \mathrm{~A}$ M Shah. ${ }^{1}$ Kings College London BHF Centre of Excellence, London, UK; ${ }^{2}$ University Hospital Maastricht, Maastricht, The Netherlands

Introduction Mice globally deficient in Nox2 are protected against cardiac fibrosis in response to chronic AngII infusion even though the degree of hypertrophy was unaltered. The selective effect of Nox2 on fibrosis may reflect its activation in a non-cardiomyocyte cell type. We hypothesised that Nox2, which is expressed in endothelial cells and inflammatory cells, may be important for cardiac fibrosis in these cell types.

Methods To investigate the role of Nox2 in inflammatory cells, we generated chimeric mice by irradiation (10Gy, $15 \mathrm{~min}$ ) to deplete resident bone marrow cells, followed by bone marrow (BM) transplantation, using the following permutations: wild-type (WT) recipient with either $\mathrm{KO}$ or WT $\mathrm{BM}$, and $\mathrm{KO}$ recipients with WT BM. To assess the role of endothelial Nox2, we used transgenic mice with endothelial-specific overexpression of Nox2 (TG) utilising the tie2 promoter construct.

Result AngII (1.1 mg/kg/day, 14-day) infusion caused similar increase in systolic hypertension and cardiac hypertrophy in all 3 chimeric groups. However, cardiac fibrosis assessed by Sirius red staining was significantly lower in KO mice receiving WT BM $(0.5 \pm 0.1 \%)$ compared to the WT:WT group $(2.7 \pm 0.7 \%)$ or in WT receiving $\mathrm{KO} \mathrm{BM}(2.3 \pm 0.6 \%)$. These data suggested that resident Nox2-expressing cells are responsible for the protective effect observed in global Nox2 KO mice. TG mice developed the same level of systolic hypertension and hypertrophy as WT littermates after AngII infusion. However, the extent of cardiac fibrosis was significantly greater in TG than WT by $\sim 2$-fold $(\mathrm{p}<0.05)$. This was associated with a greater degree of infiltration by $\mathrm{CD} 45+$ 